15

\title{
Определение электрофизических параметров полупроводника по измерениям микроволнового спектра импеданса коаксиального зонда
}

\author{
(C) А.Н. Резник, Н.К. Вдовичева
}

Институт фозики микроструктур РАН, 603950 Нижний Новгород, Россия

e-mail: reznik@ipm.sci-nnov.ru

Поступило в Редакцию 28 марта 2019 г.

В окончательной редакции 28 марта 2019 г.

Принято к публикации 15 апреля 2019 г.

\begin{abstract}
Предложен метод определения электрофизических характеристик полупроводников (концентрации и подвижности свободных носителей заряда, удельной проводимости) по данным измерений микроволнового спектра импеданса коаксиального зонда как функции приложенного постоянного напряжения $U$. Искомые параметры найдены путем решения соответствующей обратной задачи с использованием разработанной ранее теории ближнепольной антенны. Создана компьютерная программа, осуществляющая поиск решения путем минимизации многопараметрической функции невязки по алгоритму Нелдера-Мида. Точность метода проанализирована по результатам моделирования, в котором импеданс предварительно вычислен с учетом полученного профиля концентрации $n(x, U)$ обедненного слоя в окрестности контакта металлполупроводник. Продемонстрирована возможность диагностики с микронным латеральным разрешением.
\end{abstract}

Ключевые слова: микроволны, зондовая микроскопия, импеданс, полупроводник.

DOI: $10.21883 /$ JTF.2019.11.48350.150-19

\section{Введение}

Измерение вольт-фарадных характеристик - один из основных инструментов диагностики полупроводников $(C-V$-метод), позволяющий определить концентрацию свободных носителей заряда, а в более общем случае глубинный профиль концентрации. В последние годы, в связи с развитием микроволновых методов, в частности, с созданием коммерческой зондовой системы Cascade Microtech, открылась возможность локальных импедансных измерений в микроволновой части спектра [1-3]. Подобные измерения могут выполняться в условиях обеднения поверхностного слоя полупроводника за счет приложенного электрического поля $[1,3]$. Главная перспектива такой диагностики видится в реализации микронной и нанометровой латеральной разрешающей способности $C-V$-метода. Дело в том, что при диаметре емкостного контакта $<10 \mu \mathrm{m}$ чувствительность измерительного устройства в радиочастотном диапазоне оказывается недостаточной, так как измеряемая емкость $C<1 \mathrm{fF}$. С точки зрения чувствительности, микроволновые методы предпочтительнее, поскольку емкостной импеданс уменьшается обратно пропорционально частоте $f$, т.е. $X=-\operatorname{Im} Z=1 /(2 \pi f C)$. Другое важное обстоятельство заключается в том, что измерение не только мнимой, но также и действительной части импеданса $R=\operatorname{Re} Z$ (т.е. $Z-V$-спектроскопия) позволяет получить полный набор электрофизических характеристик полупроводника: концентрацию $n_{0}$, подвижность $\mu$ и тип носителей заряда, а также удельную проводимость $\sigma_{0}$.

В последние годы для локальной бесконтактной диагностики полупроводниковых материалов и структур применяется микроволновая микроскопия. Измерения выполняются в ближнем поле зонда (антенны), вследствие чего достигается субволновое пространственное разрешение, которое по порядку величины равно линейному размеру апертуры антенны. Микроскопия полупроводниковых структур в диапазоне от нескольких $\mathrm{GHz}$ до $\mathrm{THz}$ с латеральным разрешением от десятков $\mathrm{nm}$ до $100 \mu \mathrm{m}$ производилась в работах [4-9], где изучалась возможность определения проводимости $\sigma$, либо концентрации $n_{0}$. Если на микроволновый зонд дополнительно подать постоянное напряжение смещения $U$, создающее в окрестности антенны обедненный/обогащенный слой, толщина которого определяется невозмущенной концентрацией $n_{0}$, то появится возможность бесконтактно определить вышеперечисленные электрофизические параметры полупроводника. Исследования по $C-V$-диагностике полупроводников при помощи сканирующей микроволновой микроскопии нанометрового разрешения активно ведутся в последние годы [10-12]. Соответствующие методы ограничиваются определением концентрации $n_{0}$ и из-за ряда технических сложностей пока не достигли необходимой точности.

В настоящей работе дается теоретическое обоснование указанных возможностей микроволновой $Z-V$ спектроскопии полупроводников. 


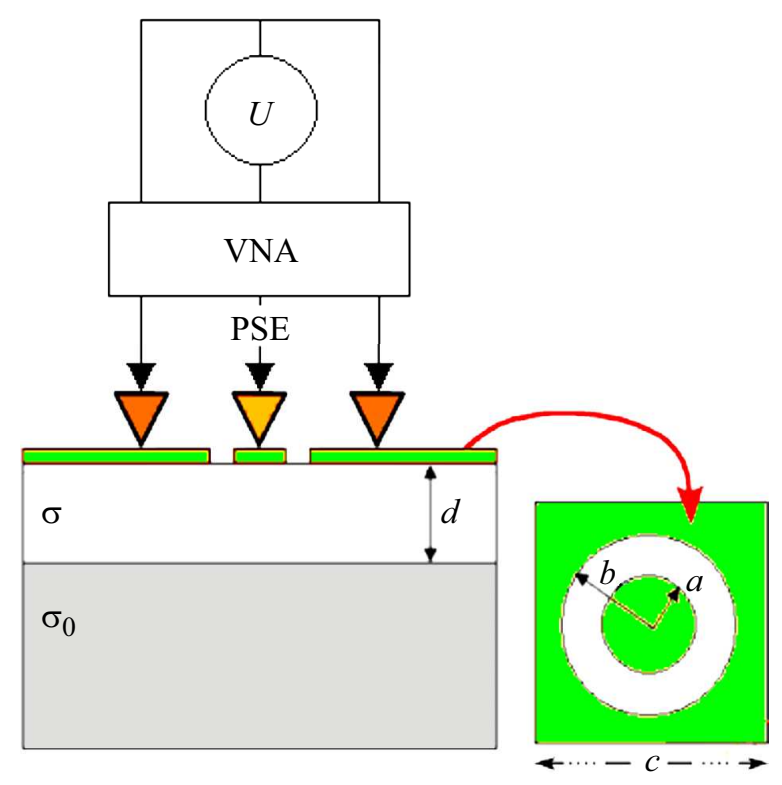

Рис. 1. Схема измерений импеданса зонда и полупроводниковая структура с контактными поверхностями антенны: VNA векторный анализатор цепей, PSE - электроды зондовой станции Cascade Microtech. На вставке справа - геометрия коаксиальной антенны.

\section{Распределение концентрации носителей в полупроводнике}

Рассмотрим коаксиальную антенну в виде концентрических металлических контактов, расположенных на поверхности образца, как показано на рис. 1. Аналогичная конфигурация антенной системы использована для измерений в [1-3]. Постоянное напряжение $U$ подается на электроды зондовой станции Cascade Microtech. Соответствующая опция имеется в некоторых серийно выпускаемых анализаторах цепей, например, Agilent E836А. После калибровки измерительной системы с использованием стандартного набора калибровочных мер измеряется микроволновый спектр комплексного импеданса антенны, как функция напряжения $Z(f, U)$. Методика подобных измерений детально описана в работах [1-3].

В рассматриваемой ситуации микроволновый импеданс коаксиальной антенны связан с глубинным профилем комплексной диэлектрической проницаемости $\varepsilon(x)=\varepsilon^{\prime}+i \sigma(x) /\left(2 \pi f \varepsilon_{0}\right)$ полученными в [6] соотношениями, где $\varepsilon_{0}-$ электрическая постоянная вакуума. Профиль проводимости выражается в виде $\sigma(x)=e \mu n(x)$, где $e-$ заряд электрона. Расчет концентрации $n(x)$, как функции напряжения $U$, может быть выполнен на основании теории контакта металлполупроводник с потенциальным барьером Шоттки [13]. Согласно этой теории, переменный ток $j$ через контакт имеет две компоненты - дрейфовую и диффузионную:

$$
j=j_{d r}+j_{d i f}=-e n \mu \frac{d \varphi}{d x}+\mu \kappa T \frac{d n}{d x},
$$

где $\varphi(x)$ - распределение потенциала в полупроводнике, $T-$ температура, $\kappa-$ постоянная Больцмана. Граничные условия для $\varphi$ и $n$ имеют вид: $\varphi(x=0)=0$, $\varphi(x \rightarrow \infty)=U_{k}+U, n(x \rightarrow \infty)=n_{0}$. Здесь $U_{k}-$ барьерная разность потенциалов, которая возникает на контакте при $U=0$. В предположении $j=0$ из (1) получим равновесный профиль концентрации

$$
n(x)=n_{0} \exp \left\{\frac{e}{\kappa T}\left[\varphi(x)-\left(U_{k}+U\right)\right]\right\} .
$$

Возможность использовать соотношение (2) в отсутствие равновесия, когда $j \neq 0$, требует отдельного исследования, которое выполнено далее. Учитывая соотношение (2), из уравнения Пуассона получаем уравнение для потенциала

$$
\frac{d^{2} \varphi}{d x^{2}}=\frac{e n_{0}}{\varepsilon^{\prime} \varepsilon_{0}}\left\{\left[\exp \frac{e\left(\varphi-\left(U_{k}+U\right)\right)}{\kappa T}\right]-1\right\} .
$$

Решая уравнение (3) и используя (2), получим искомый профиль концентрации $n(x, U)$. В частности, из (3) получается решение для случая сильного обеднения, когда при достаточно большом смещении $U_{k}+U \gg \kappa T / e$ для обедненного слоя толщины $d$ имеем $n(x) \ll n_{0}-$ основной рассматриваемый нами режим. В такой ситуации получаем приближенное решение [13]

$$
\varphi(x)=\left\{\begin{array}{ll}
-\frac{e n_{0}}{2 \varepsilon^{\prime} \varepsilon_{0}}(d-x)^{2}+\left(U_{k}+U\right), & x \leq d \\
U_{k}+U, & x>d
\end{array},\right.
$$

где

$$
d=\sqrt{\frac{2 \varepsilon^{\prime} \varepsilon_{0}\left(U_{k}+U\right)}{e n_{0}}}
$$

есть функция невозмущенной концентрации и напряжения. В настоящей работе мы не конкретизируем тип основных носителей заряда в полупроводнике. Как видно из формулы (5), толщина $d$ растет с увеличением напряжения при $U>0$. Если под $U$ понимать напряжение смещения на центральном контакте антенны, такая ситуация соответствует полупроводнику $p$ типа. Тогда для $n$-полупроводника в формулах (2)-(5) следует сделать замену $U \rightarrow-U$. Отметим, что профиль концентрации под центральным контактом в геометрии измерений на рис. 1 можно считать одномерным только в том случае, когда площади внешнего и центрального контактов находятся в соотношении $S_{b} \gg S_{a}$, т. е. если $b, c \gg a$. Именно такое соотношение размеров реализовано в измерениях [1-3] при $a=5-25 \mu \mathrm{m}$. В этих условиях напряжение $U$ падает на центральный контакт, по крайней мере, при $U>-U_{k}$, пока под этим контактом существует обедненный слой, что позволяет 

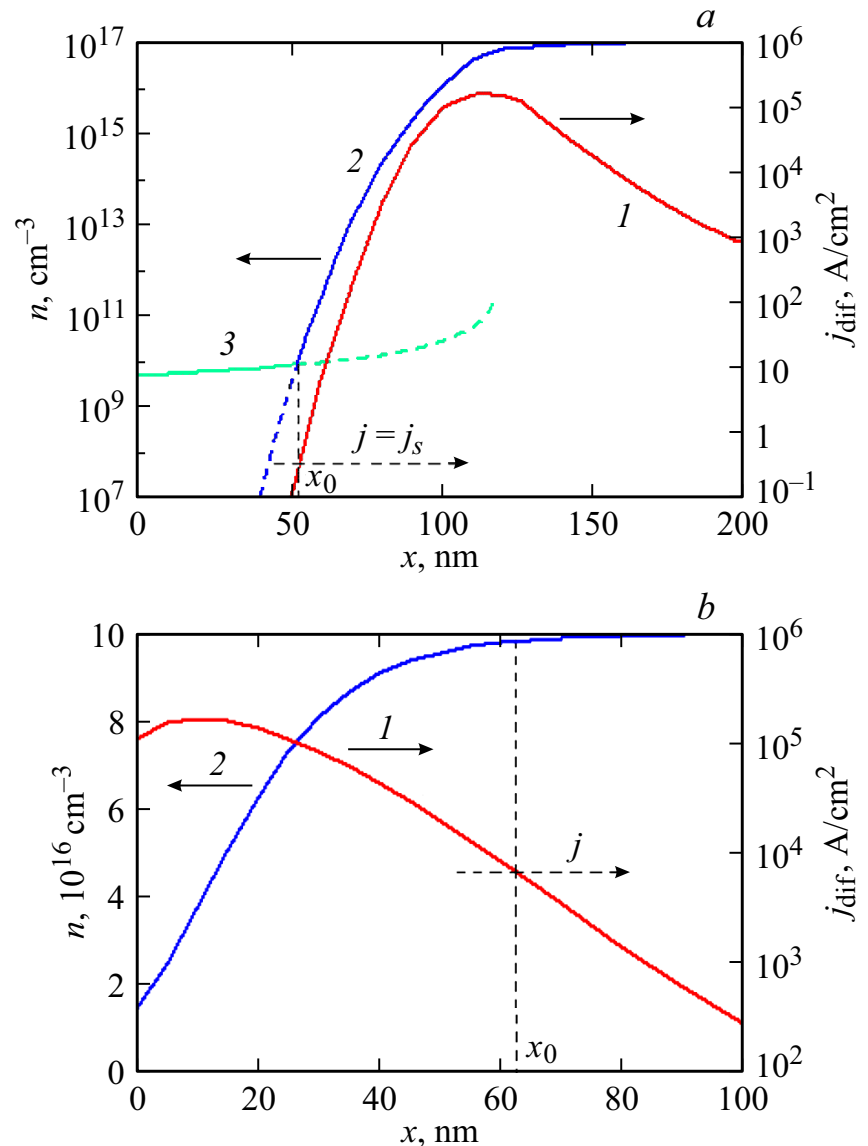

Рис. 2. Диффузионный ток (кривые 1 ) и концентрация носителей заряда (кривые 2,3), как функции координаты $x$ в глубине полупроводника. Кривые 2 - расчет по уравнениям (2),(3), кривая 3 - расчет по формуле (6). $a$ : $U=1 \mathrm{~V}-$ сильное обеднение; $b$ : $U=-0.25 \mathrm{~V}$ - слабое обеднение.

использовать граничное условие $\varphi(x \rightarrow \infty)=U_{k}+U$ и соотношения (2)-(5) для концентрации $n(x, U)$.

При подстановке $\varphi^{\prime}=\Psi(\varphi)$ уравнение (3) интегрируется:

$$
x=A \int_{\varphi}^{\frac{U+U_{k}}{\kappa T}} \frac{d \eta}{\sqrt{2\left(e^{\eta}-\eta-1\right)}},
$$

где коэффициент

$$
A=\operatorname{sign}\left(U+U_{k}\right) / \sqrt{\frac{e n_{0}}{\varepsilon^{\prime} \varepsilon_{0} \kappa T}} .
$$

Для получения функции $\varphi(x)$ этот интеграл вычисляется методом трапеций, а концентрация $n(x, U)$ рассчитывается по формуле (2). Нами создана компьютерная программа, реализующая указанный алгоритм расчета функции $n(x, U)$.

Возьмем для примера $U_{k}=0.3 \mathrm{~V}, n_{0}=10^{17} \mathrm{~cm}^{-3}$ и в приконтактной области при $U=0$ возникает обедненный слой толщиной $d_{0}=58 \mathrm{~nm}$ согласно (5). Профиль $n(x, U=1 \mathrm{~V})$ при $T<300 \mathrm{~K}, \mu=1.5 \cdot 10^{3} \mathrm{~cm}^{2} /(\mathrm{Vs})$, $\varepsilon^{\prime}=10$ представлен на рис. 2, $a$. В этом случае имеем $d=121 \mathrm{~nm}$. На рис. 2, $a$ кривой 1 показан диффузионный ток в (1) $j_{d i f}=\mu \kappa T d n / d x$. Приближенное соотношение (2) и вытекающее из него уравнение (3) справедливы при условии $j_{d r} \approx j_{d i f} \gg j$, где дрейфовый ток $j_{d r}$ - первое слагаемое в (1). Указанное условие объясняется так же, как и равновесное приближение $n(0) \approx n_{0} \exp \left(-e U_{k} / \kappa T\right)$ при $|U| \ll U_{k}$ в [13 (стр. 218)]. При сильном обеднении имеет место насыщение тока $j=j_{s}=(1 / 4) e v_{t} n_{0} \exp \left(-e U_{k} / \kappa T\right)$ [13], где $v_{t}-$ тепловая скорость свободных электронов. Принимая $v_{t} \approx 10^{7} \mathrm{~cm} / \mathrm{s}$, получаем $j_{s}=0.37 \mathrm{~A} / \mathrm{cm}^{2}$. Как можно видеть из рис. 2, $a$, указанное условие выполняется в области $x>x_{0} \approx 50 \mathrm{~nm}$, где градиент концентрации $n(x)$ достаточно велик. При $x<x_{0}$ уравнения (2), (3) не применимы. В этой области $j_{d i f} \rightarrow 0, j_{d r} \approx j_{s}$, т.е. имеет место более точное решение для профиля концентрации:

$$
n(x)=\frac{j_{s} \varepsilon^{\prime} \varepsilon_{0}}{e^{2} \mu n_{0}} \frac{1}{d-x},
$$

которое получается из уравнения (1) при $j_{d i f}=0$ с учетом (4). Решение (6) показано на рис. 2, а линией 3. В рассматриваемом примере разделяющей границей, при которой решения (2), (3) и (6) дают одинаковое значение концентрации $n\left(x=x_{0}\right)$, является координата $x_{0}$. При $U<0$ имеет место резкое возрастание тока $j$. Тем не менее уравнения (2),(3) адекватно описывают профиль $n(x)$ в области большого градиента концентрации. Соответствующий пример для $U=-0.25 \mathrm{~V}$ представлен на рис. 2, $b$. При $-U_{k}<U<0$ ток растет, как $j(U)=j_{s} \exp (-e U / \kappa T)$ [13], т.е. в рассматриваемом примере $j=6.4 \cdot 10^{3} \mathrm{~A} / \mathrm{cm}^{2}$. Тем не менее условие $j_{d r} \approx j_{d i f} \gg j$ выполняется и в этом случае при $x<x_{0} \approx 63 \mathrm{~nm}$, т.е. там, где $n(x)$ наиболее сильно отличается от $n_{0}$, что ясно видно из рис. $2, b$. При $x>x_{0}$ имеют место несушественные для дальнейшего исследования поправки к профилю $n\left(x>x_{0}\right) \approx n_{0}$.

\section{Решение обратной задачи}

Полученный из уравнений $(2),(3),(6)$ профиль $n(x)$ использован для расчета проводимости $\sigma(x)$ и комплексной проницаемости $\varepsilon(x)$ полупроводника с обедненным поверхностным слоем за счет приложенного к коаксиальным контактам напряжения $U$. В рамках рассматриваемых приближений мы вычисляем частотный спектр импеданса коаксиальной антенны $Z=R+i X$, как функцию $U$, используя теорию [6]. Возможность применения теории, построенной для плоскослоистой среды, в геометрии обедненного (изолирующего) слоя, существующего только в области центрального контакта коаксиала радиуса $a$, показана в [2]. Точность соответствующего описания характеризуется краевым эффектом на этом контакте, который, согласно [2], не превышает 1-3\%. Когда размеры антенны на рис. 1 характеризуются соотношением $b, c \gg a$, в расчетах импеданса достаточно 

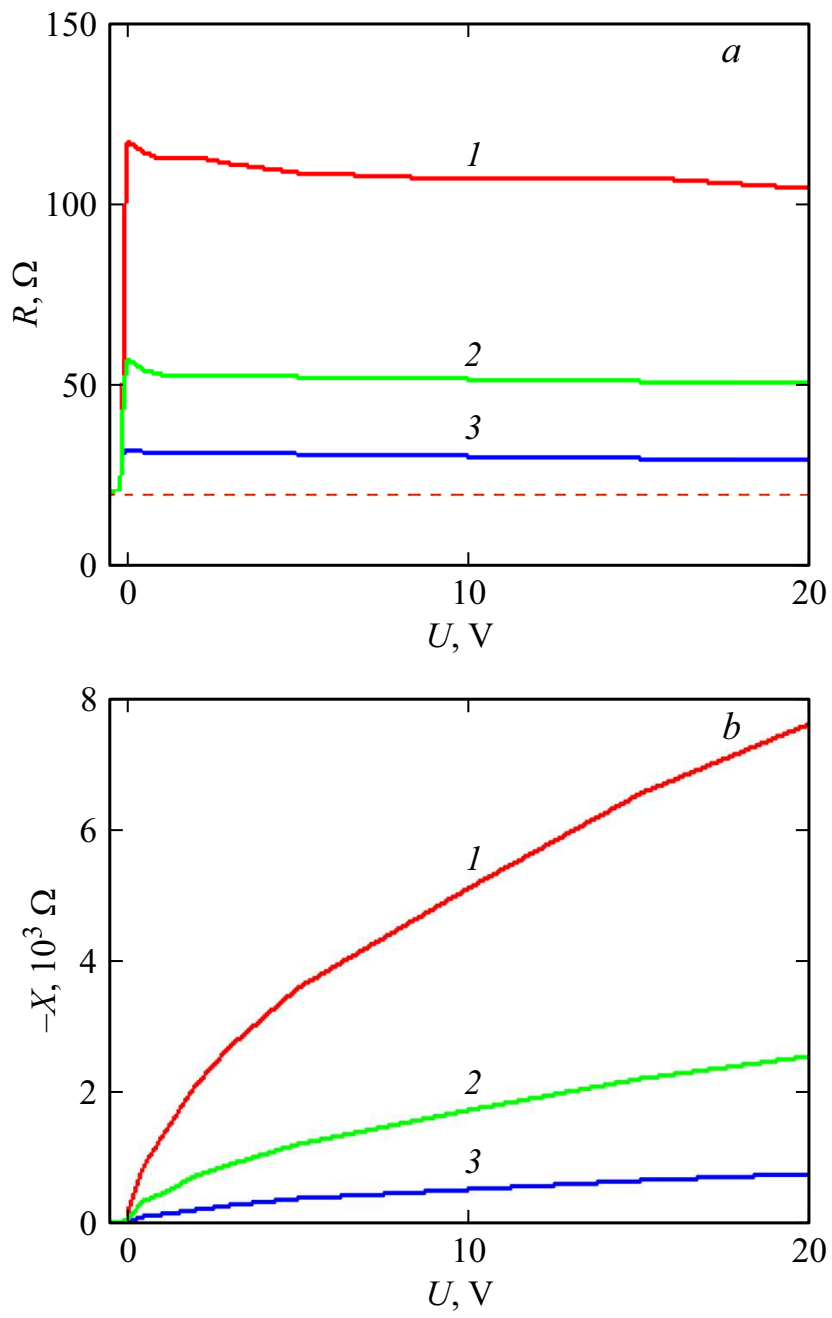

Рис. 3. Зависимость импеданса $Z$ от напряжения смещения $U$ на центральном контакте. $a$ - сопротивление $R=\operatorname{Re} Z ; b-$ реактанс $X=\operatorname{Im} Z$. Кривые $1-3$ соответствуют частоте $f=1,3,10 \mathrm{GHz}$. Штриховая линия - импеданс $Z \approx 20.5-i 0-$ для однородно легированного полупроводника $n(x)=n_{0}=$ const.

учесть только центральный контакт. В этом случае антенна рассматривается, как электрический монополь, излучающий квазистатическое электрическое поле [6]. Результаты расчета зависимости $Z(U)$ для нескольких частот $f$ при $a=5.5 \mu \mathrm{m}$ представлены на рис. 3. Использованы приведенные выше значения параметров полупроводника, для которых имеем невозмущенную проводимость $\sigma_{0}=e \mu n_{0}=24(\Omega \mathrm{cm})^{-1}$. Опыт выполненных в [2] измерений зондовой станцией Cascade Microtech показал хорошую точность в диапазоне изменения импеданса $5 \Omega<R,-X<10 \mathrm{k} \Omega$. Приведенные на рис. 3 значения $R,-X$ не выходят за указанные пределы. Таким образом, предлагаемый метод диагностики может быть реализован при микронном разрешении антенны, которое в рассматриваемом случае определяется радиусом $a$ центрального контакта (рис. 1).
Полученные значения $Z(f, U)$ (рис. 3) в выполненном компьютерном моделировании использовались в качестве „экспериментальных“ данных $Z=Z_{e}$ в процессе решения обратной задачи. Поиск решения производился для упрощенной модели обедненного слоя в виде однородной пленки толщины $d$ и проводимости $\sigma$, расположенной поверх однородного полупространства с невозмущенной проводимостью $\sigma_{0}$, как показано на рис. 1. Обратная задача заключалась в поиске компонент вектора $\mathbf{G}=\left\{d, \sigma, \sigma_{0}\right\}$ по данным „измерений“ импеданса $Z_{e}$ на нескольких частотах $f_{k}(k=1,2, \ldots N)$ при фиксированном напряжении $U$. Процедура повторялась для нескольких $(\sim 10-15)$ значений $U$ из диапазона $-0.25<U<20 \mathrm{~V}$. Концентрация носителей $n_{0}$ определялась с использованием полученной зависимости $d(U)$ и формулы (5), отвечающей рассматриваемой приближенной модели полупроводника с обедненным слоем.

Решение обратной задачи находилось путем минимизации функции невязки:

$$
\begin{aligned}
F[\mathbf{G}(U)] & =\sum_{k=1}^{N}\left\{\frac{\left[R_{e}\left(f_{k}, U\right)-R\left(\mathbf{G}, f_{k}, U\right)\right]^{2}}{R_{e}\left(f_{k}, U\right)^{2}+X_{e}\left(f_{k}, U\right)^{2}}\right. \\
& \left.+\frac{\left[X_{e}\left(f_{k}, U\right)-X\left(\mathbf{G}, f_{k}, U\right)\right]^{2}}{R_{e}\left(f_{k}, U\right)^{2}+X_{e}\left(f_{k}, U\right)^{2}}\right\}^{1 / 2},
\end{aligned}
$$

где $Z(\mathbf{G}, f, U)=R(\mathbf{G}, f, U)+i X(\mathbf{G}, f, U)$ - результат расчета импеданса $Z$ для произвольного набора компонент вектора $\mathbf{G}$, начиная с выбранного начального значения $\mathbf{G}=\mathbf{G}_{0}$. В качестве решения обратной задачи принимался вектор $\mathbf{G}=\mathbf{G}_{\min }$, для которого функция (7) достигает минимума.

Как правило, $F(\mathbf{G})$ имеет овражную структуру, и минимизация таких функций от большого количества искомых параметров (компонент вектора $\mathbf{G}$ ) является сложной задачей. Овражная структура функции невязки характеризуется поведением этой функции вблизи точки $\mathbf{G}_{\min }$ :

$$
\begin{aligned}
F(\mathbf{G}) \approx & F\left(\mathbf{G}_{\min }\right)+\frac{1}{2}\left(H\left(\mathbf{G}_{\min }\right)\left(\mathbf{G}-\mathbf{G}_{\min }\right)\right) \\
& +O\left(\left\|\mathbf{G}-\mathbf{G}_{\min }\right\|^{3}\right) .
\end{aligned}
$$

Здесь

$$
H(\mathbf{G})=\left\{\frac{\partial^{2} F}{\partial G_{i} \partial G_{j}}\right\}
$$

- матрица Гессе $(i, j=1, \ldots m$, где $m$ - число компонент вектора $(\mathbf{G})), \mu(H)$ - собственные числа матрицы $H$, а запись $(\mathbf{u}, \mathbf{v})$ означает скалярное произведение векторов. Если число обусловленности

$$
B=\frac{\max \mu(H)}{\min \mu(H)} \gg 1,
$$

можно утверждать, что $F(\mathbf{G})$ плохо минимизируется. Проблема может возникнуть даже при малом числе параметров минимизации. Чтобы обойти эту трудность, 


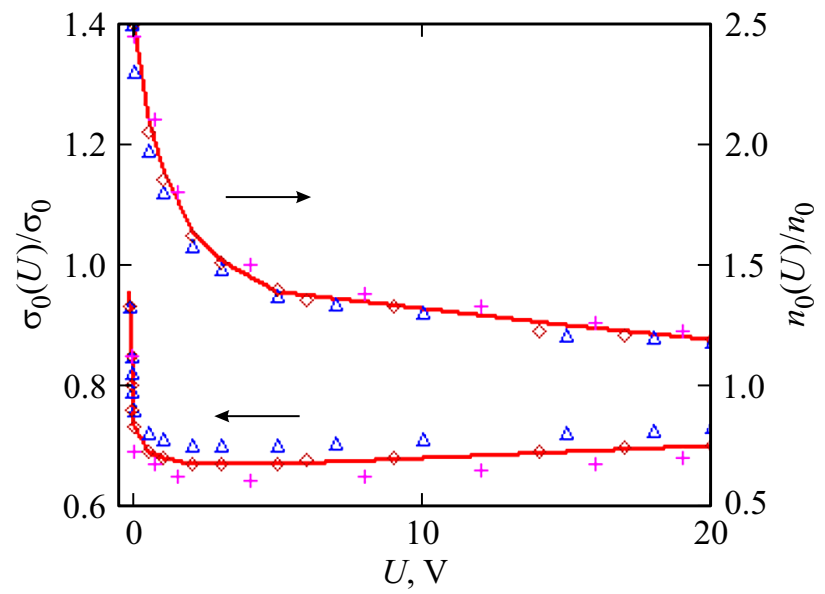

Рис. 4. Решение обратной задачи. Линии - средние значения, значки - решения для различных наборов частот $f$.

мы использовали метод Нелдера-Мида, известный, как метод деформируемого многогранника или симплекс метод [14]. Идея метода следующая: $m$-симплекс это выпуклый многогранник с вершинами в $m+1$ точках, не лежащих в одной плоскости в $m$-мерном евклидовом пространстве. Мы сравниваем значения функции невязки $F(\mathbf{G})$ в вершинах первоначального симплекса и трансформируем симплекс в направлении оптимальной точки, применяя итеративную процедуру. Симплекс трансформируется с использованием трех основных операций: отражение, расширение, сужение. Это достоверный метод прямой минимизации функции невязки, сходимость которого доказана для числа переменных $m \leq 6$. Программа, созданная на основе метода НелдераМида, показала хорошую сходимость для поиска трех вышеуказанных компонент вектора $\mathbf{G}$.

Результаты решения обратной задачи в виде зависимостей $n_{0}(U) / n_{0}, \sigma_{0}(U) / \sigma_{0}$ показаны на рис. 4 для „измерений“ на нескольких частотах диапазона $f=1-10 \mathrm{GHz}$. Полученные значения $n_{0}, \sigma_{0}$ оказались зависящими от напряжения $U$ в связи с тем, что при поиске решения обратной задачи использована приближенная модель профиля $n(x, U)$ со скачкообразным изменением концентрации при $x=d$. В модельных расчетах „экспериментальных“ значений импеданса $Z_{e}$ формулы (7) взяты более реалистичные непрерывные профили $n(x, U)$, показанные на рис. 2. Отметим, что минимальный набор частот $N=2$ позволяет найти решение рассматриваемой задачи определения трех параметров, если на каждой частоте измеряются сопротивление $R$ и реактанс $X$ антенны. При этом частоты не должны быть слишком близкими. Например, при $f_{1}=1-3 \mathrm{GHz}$, $f_{2}=5-10 \mathrm{GHz}$ получим искомое решение. Увеличение количества частот $N \geq 3$ приводит к незначительному изменению решения, как это видно из рис. 4. Можно также видеть, что с ростом напряжения $U$ полученная концентрация $n_{0}(U)$ стремится к принятому в модели точному значению $n_{0}$. При $U>15 \mathrm{~V}$ точность решения для $n_{0}$ оказалась вполне приемлемой $\sim 20 \%$. Для $\sigma_{0}$ наилучшая точность $\sim 10-20 \%$ достигается при небольшом отрицательном смещении $-U \approx 0.1-0.15 \mathrm{~V}$. Используя полученные значения $n_{0}(U), \sigma_{0}(U)$, находим подвижность $\mu=\sigma_{0} /\left(e n_{0}\right)$, которая при $2<U<10 \mathrm{~V}$ с точностью $\sim 5-10 \%$ соответствует принятому в расчетах значению. Как сказано выше, погрешность метода, присущая также и классическим $C-V$-измерениям, связана с тем, что при решении обратной задачи использовалась достаточно грубая модель для профиля $n(x, U)$, показанного на рис. 2. Точность может быть повышена, если учесть в модели довольно протяженный непрерывный переход от пониженной к невозмущенной концентрации.

Эффективная проводимость $\sigma$ обедненного слоя также определяется в процессе решения обратной задачи и составляет в рассматриваемом случае $\sim(1-5) \cdot 10^{-4}(\Omega \mathrm{cm})^{-1}$, что, как и следует ожидать, на несколько порядков меньше невозмущенной концентрации $\sigma_{0}$. Тем не менее проводимость $\sigma$ существенно влияет на сопротивление антенны $R$, что можно видеть из рис. 3,a. Полагая для обедненного слоя $\sigma=0$, получим сопротивление $R \approx 20.5 \Omega$, которое в нашем примере не зависит от частоты $f$ и толщины $d$, а определяется только проводимостью $\sigma_{0}$ невозмущенной области и радиусом антенны $a$. Указанное значение $R$ есть сопротивление растекания переменного тока в области полупроводника под запорным слоем. Заметим, что для определения параметров $n_{0}, \sigma_{0}, \mu$ полупроводника не требуется находить проводимость $\sigma$. Однако зависимость $\sigma(U)$ может представлять интерес для изучения физики процесса обеднения. Например, можно исследовать эффект баллистического пролета носителей через область резкого изменения концентрации $n(x)$ на границе обедненной области в толще полупроводника. Соответствующий эффект не учитывался в дрейфоводиффузионной модели (2)-(5), но может быть важен, когда толщина $\Delta x$ переходной области близка к длине свободного пробега носителей. В примерах на рис. $2 \Delta x$ составляет 30-40 nm. С точки зрения такого исследования важно решить проблему восстановления непрерывного профиля $n(x, U)$ с более адекватным описанием переходной области, что, как было сказано, повысит также и точность определения основных параметров.

\section{Заключение}

В работе предложен метод, который позволяет с микронным латеральным разрешением определить концентрацию и подвижность носителей заряда в полупроводнике по данным микроволновых измерений спектра комплексного импеданса коаксиального зонда. Дальнейшее развитие метода может быть связано с исследованием полупроводниковых структур - пленочных, селективно легированных, транзисторных с проводящим каналом и др. Результаты работы [2] дают основания полагать, что метод может быть реализован с использованием 
антенной решетки, сформированной непосредственно на поверхности образца. Возможность аналогичных бесконтактных измерений при помощи сканирующего микроволнового микроскопа требует отдельного исследования.

\section{Финансирование работы}

Работа поддержана Российским фондом фундаментальных исследований, грант № 18-02-00914.

\section{Конфликт интересов}

Авторы заявляют, что у них нет конфликта интересов.

\section{Список литературы}

[1] Vostokov N.V., Shashkin V.I. // IEEE Tr. Electron. Dev. 2017. Vol. 64. N 1. P. 109-114.

[2] Reznik A.N., Vostokov N.V., Vdovicheva N.K., Korolyov S.A., Shashkin V.I. // J. Appl. Phys. 2017. Vol. 122. P. 244505: 1-9.

[3] Vostokov N.V., Koblov E.A., Korolyov S.A., Revin M.V., Shashkin V.I. // IEEE Tr. Electron Dev. 2018. Vol. 65. N 4. P. 1327-1332.

[4] Imtiaz A., Baldwin T., Nembach H.T., Wallis T.M., Kabos P. // Appl. Phys. Lett. 2007. Vol. 90. P. 23105: 1-3.

[5] Laji K., Kundhikanjana W., Kelly M.A., Shen Z.-X. // Appl. Nanosci. 2011. P. 13-18.

[6] Reznik A.N., Korolyov S.A. // J. Appl. Phys. 2016. Vol. 119. P. 094504: 1-10.

[7] Korolyov S.A., Reznik A.N. // Rev. Sci. Instrum. 2018. Vol. 89. P. 023706: 1-9.

[8] Buersgens F., Kersting R., Chen H.-T. // Appl. Phys. Lett. 2006. Vol. 88. P. 112115: 1-3.

[9] Трухин В.Н., Голубок А.О., Лютецкий А.В., Матвеев Б.А., Пихтин Н.А., Самойлов Л.Л., Сапожников И.Д., Тарасов И.С., Фельштын М.Л. // Изв. вузов. Радиофизика. 2011. T. 54. № 89. C. 640-648.

[10] Huber H.P., Humer I., Hochleitner M., Fenner M. et al. // J. Appl. Phys. 2012. Vol. 111. P. 014301: 1-10.

[11] Amster O., Stanke F., Friedman S., Yang Y., DixonWarren St.J., Drevniok B. // Microelectron. Reliab. 2017. Vol. 76-77. P. 214-217.

[12] Hommel S., Killat N., Altes A., Schveinboeck T., Kreupl F. // Microelectron. Reliab. 2017. Vol. 76-77. P. 218-221.

[13] Бонч-Бруевич В.Л., Калашников С.Г. // Физика полупроводников. М.: Мир, 1977. $678 \mathrm{c.}$

[14] Nelder J.A., Mead R. // Comput. J. 1965. Vol. 7. P. 308-313. 